\section{Formulação da Política de Gestão do Trabalho e Educação na Saúde: o caso da Secretaria Estadual de Saúde da Bahia, Brasil, 2007-2008}

\author{
Management Policy-Making for Work and Education \\ in Health: the case of the Bahia State Health \\ Department, Brazil, 2007-2008
}

\author{
1 Instituto de Saúde Coletiva \\ Universidade Federal da \\ Bahia, Salvador, Brasil. \\ Correspondência \\ I. C. M. Pinto \\ Instituto de Saúde Coletiva \\ Universidade Federal da \\ Bahia. \\ Rua Basílio da Gama s/n, \\ Salvador, $B A$ \\ 40110-040, Brasil. \\ isabela@ufba.br
}

\begin{abstract}
The construction of Brazil's Unified National Health System (SUS) has raised a set of challenges for the health sector's administrators and personnel, including issues of work management and continuing education for health workers, in view of the financial, political, and organizational constraints in the process of changing the healthcare model. The current study aimed to analyze the process of formulating the Health Work and Education Management Policy by the Bahia State Health Department. Public policy cycle was used as the theoretical framework. The study analyzed data from institutional documents and records of participant observation by one of the authors. The results include mapping the governmental and nongovernmental stakeholders that participated in the process. The analysis highlights a series of problems in the SUS in Bahia related to work management and health workers' profile, taken as the point of departure for prioritysetting in the State Strategic Agenda and Health Plan for 2008-2011.
\end{abstract}

Health Public Policy; Health Personnel; Unified Health System
Isabela Cardoso de Matos Pinto 1

Carmen Fontes Teixeira 1

\section{Introdução}

O sistema público de saúde brasileiro vem passando, nos últimos 22 anos, por um processo de reforma voltado à construção do Sistema Único de Saúde (SUS), cujas bases políticas e doutrinárias foram estabelecidas na Constituição Federal de 1988. Nesta, o direito à saúde é direito de cidadania, correlato à responsabilidade estatal pela garantia da universalidade e integralidade no acesso da população às ações e serviços.

A trajetória institucional de construção do SUS tem assumido ritmos e formas diferenciadas em cada Unidade da Federação, na medida em que se adotou a municipalização das ações e serviços durante a década de 90 (Normas Operacionais Básicas - NOB 01/91; 01/93; 01/96) 1,2 e, posteriormente, a regionalização dos serviços de saúde (Norma Operacional de Assistência à Saúde - NOAS 2001-2001), visando a promover a organização do modelo de atenção com base no princípio da integralidade do cuidado 3,4. A partir de 2006, o desenvolvimento do SUS passou a se fundamentar no Pacto pela Vida, em defesa do SUS e de gestão 5 , documento de orientação e condução político-gerencial, aprovado pela Comissão Intergestores Tripartite, reforçando-se a regionalização dos serviços e a proposta de constituição de redes integradas que articulassem os diferentes níveis de atenção.

No balanço do processo de construção do SUS, constatam-se avanços e dificuldades na 
operacionalização dos seus princípios e diretrizes. Isso tem estimulado a mobilização de vários atores políticos que defendem sua implementação, bem como o levantamento de questões e a realização de estudos acerca dos fatores que influenciam o processo de formulação e implementação das políticas públicas em geral e da política de saúde em particular. Sendo assim, vêm se problematizando os arranjos políticos e operacionais que, em cada estado e/ou município, determinam o avanço ou recuo da incorporação dessas propostas no SUS, em especial no que se refere à descentralização dos recursos, do poder decisório e das responsabilidades sobre a organização e a produção dos serviços 6,7,8,9.

Um dos aspectos que tem chamado, particularmente, a atenção dos gestores e pesquisadores é a questão dos recursos humanos, considerada um dos "nós críticos" do processo de mudança da gestão e da atenção à saúde no país. De fato, a escassez de quadros qualificados para o exercício da gestão dos sistemas e serviços, além da precarização das relações de trabalho, somadas à inadequação dos perfis e ausência de compromisso de grande parte dos profissionais e trabalhadores de saúde para com o SUS, constituem, hoje, um dos grandes desafios à sua consolidação.

O enfrentamento dessas dificuldades vem gerando a elaboração de várias propostas de soluções e arranjos para a gestão dos trabalhadores da saúde, especificamente no âmbito estadual e municipal, por conta do processo de descentralização. Se, por um lado, isso facilita a definição de propostas mais adequadas à problemática regional e local, por outro, tem evidenciado a existência de problemas estruturais e conjunturais, que repercutem na capacidade de gestão de muitas secretarias de estado e municípios na área de gestão do trabalho e da educação em saúde.

Considerando que essa área é estratégica, visto que o investimento e a priorização do seu desenvolvimento institucional podem vir a desencadear mudanças nas práticas político-institucionais tanto no âmbito gerencial quanto na organização e prestação dos serviços, é importante resgatar a experiência que vem sendo desenvolvida pelas secretarias de saúde. Logo, cabe destacar o esforço desenvolvido pela Secretaria Estadual de Saúde da Bahia (SESAB) no redirecionamento do processo de implantação do SUS no estado, na conjuntura posterior às eleições de outubro de 2006

De fato, no cenário político configurado na Bahia a partir de janeiro de 2007, o grupo dirigente adotou como eixos norteadores do governo a ampliação da democracia na saúde, a gestão participativa e a negociação coletiva em torno das estratégias de desenvolvimento eco- nômico e social 10. A equipe gestora da SESAB assumiu, entre outros compromissos, a superação da precarização das relações e condições de trabalho, bem como a remuneração dos trabalhadores da saúde; a organização dos serviços e atuação dos profissionais de saúde; a responsabilização de gestores, gerentes e profissionais para prestar o melhor cuidado possível dentro das condições limitadas em termos financeiros e operacionais 10 .

A área de recursos humanos, portanto, foi considerada prioridade, o que se traduziu na formulação da Política de Gestão do Trabalho e Educação na Saúde (PGETES), desenvolvida sob a coordenação da Superintendência de Recursos Humanos da SESAB. Investigar como se deu esse processo pode contribuir para que se compreenda a atuação do governo em uma situação na qual se busca estimular a participação política de distintos atores.

Nessa perspectiva, este trabalho tem como objetivos: (a) identificar e caracterizar os atores políticos que participaram do processo de formulação da PGETES; (b) descrever e analisar os momentos desse processo; (c) discutir algumas das limitações encontradas e as possibilidades de aperfeiçoamento da prática de formulação e implementação de políticas no âmbito estadual.

\section{Procedimentos teórico-metodológicos}

Foi realizado um estudo de caso que teve como objeto o processo de formulação da PGETES no âmbito da SESAB, no período 2007-2008. As informações obtidas foram sistematizadas e analisadas, tomando-se como referencial a teoria do ciclo da política de Kingdon 11, adaptada à análise do processo de formulação de políticas de saúde por Teixeira et al. 12 .

A literatura do ciclo de política tem adquirido progressiva importância nos estudos sobre os processos de formulação e implementação de políticas no âmbito governamental.Viana 13 identifica vários trabalhos que indicam os estágios de desenvolvimento das políticas, quais sejam: (a) determinação da agenda, isto é, definição do(s) problema(s) que se tornam objeto de política pública; (b) formulação e legitimação da política (seleção de propostas, construção de apoio político, formalização das decisões em documentos como leis, decretos, planos, programas, portarias etc.); (c) implementação das políticas (operacionalização em planos, programas e projetos no âmbito da burocracia e sua execução); (d) avaliação de políticas (análise dos resultados alcançados com a implementação, impactos e sugestão de mudanças). 
Dos autores que enfatizam a análise da determinação da agenda e da formulação da política, destacam-se Baumgartner \& Jones 14 e Kingdon 11. Ambos os trabalhos tomam como ponto de partida a compreensão de que o processo decisório envolve sempre duas questões: onde surge a demanda e quem participa do processo, chamando a atenção para o fato de que um assunto ou tema, para atingir o status de agenda, precisa ser alvo de atenções dos atores que participam da cena política. Tal processo envolve a interação de uma série de elementos complexos, que podem ser identificados e analisados em distintos momentos das tomadas de decisão.

Nesse sentido, Kingdon 11 elaborou um modelo analítico que contempla a descrição dos momentos e dos elementos que compõem o processo: os momentos de "pré-decisão" e de "decisão" e os atores, arenas, problemas e alternativas de políticas que se constituem em objeto do processo decisório. Esse modelo vem sen- do utilizado no Brasil em trabalhos como o de Pinto 15, que analisa a ascensão de questões na agenda governamental da área de saúde, especificamente a proposta de criação das organizações sociais como alternativas de gestão de unidades de produção de serviços, a exemplo dos hospitais públicos.

Assumindo essa perspectiva, decidimos trabalhar com base na adaptação feita por Teixeira et al. 12 do modelo analítico de Kingdon 11, buscando aproximá-lo do processo de formulação e implementação da PGETES (Figura 1). A Figura proposta contempla, assim, múltiplos aspectos envolvidos na análise do processo decisório ocorrido no âmbito da Superintendência de Recursos Humanos da SESAB, no período 2007-2008, orientando o processamento das informações relativas a três grandes categorias de análise: o contexto, os atores e o processo decisório.

A caracterização do contexto implica distinguir, do ponto de vista analítico, o contexto

Figura 1

Modelo teórico para análise do processo de formulação da Política de Gestão do Trabalho e Educação na Saúde (PGETES).

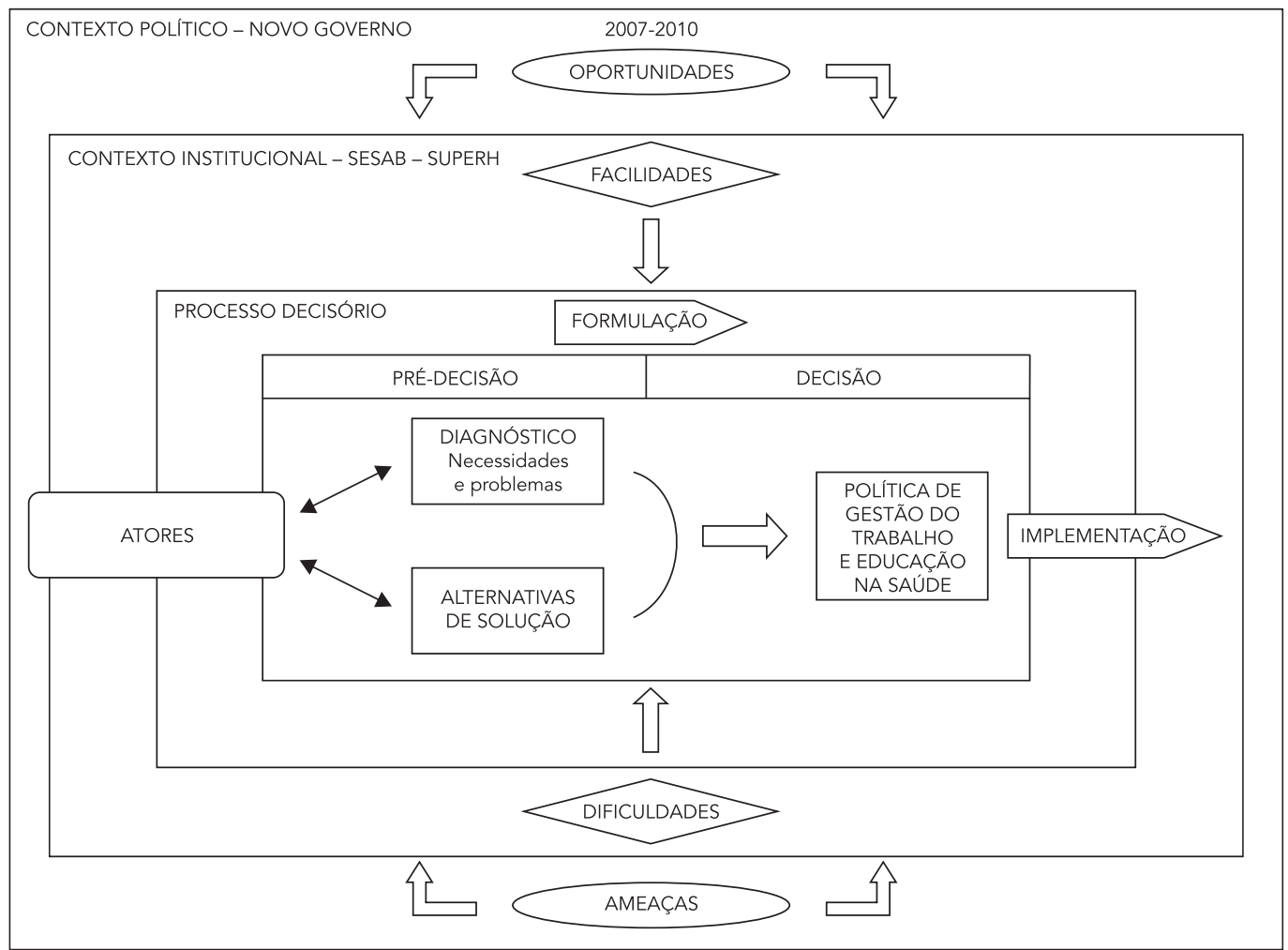

SESAB: Secretaria Estadual de Saúde da Bahia; SUPERH: Superintendência de Recursos Humanos.

Nota: adaptação feita por Teixeira et al. 12, com base na proposta de Kingdon 11. 
político mais geral do Estado da Bahia do contexto político-institucional, isto é, a SESAB. Esta configura o espaço para onde confluem as diretrizes adotadas pelo Governo Estadual e as determinações oriundas do processo de reforma do sistema público de saúde, sabidamente uma política de estado ancorada na Constituição Federal e na legislação do SUS.

Quanto aos atores, este referencial permite, em primeiro lugar, que se distingam os atores institucionais responsáveis pela gestão das políticas e programas da área de gestão do trabalho e educação na saúde na SESAB, notadamente os dirigentes e técnicos dos órgãos que compõem a Superintendência de Recursos Humanos, dos demais atores institucionais, particularmente os dirigentes das outras superintendências (que apresentam demandas à área de recursos humanos) e de órgãos responsáveis pelo planejamento na instituição, isto é, pela formulação da agenda e das políticas de saúde estaduais. Em segundo lugar, permite que se identifiquem, entre os participantes do processo, outros atores governamentais e não governamentais que atuam em instituições vinculadas à problemática de recursos humanos em saúde, como universidades, organizações representativas dos profissionais e trabalhadores de saúde que possam ter se envolvido com a formulação da PGETES, bem como outros atores, representantes dos usuários do sistema ou membros de organizações nacionais e internacionais que possam ter atuado como consultores da política.

No que diz respeito ao desenvolvimento do processo propriamente dito, esse diagrama orienta a organização e análise das informações relativas aos momentos da pré-decisão e da decisão. Permite a identificação dos problemas e das propostas (alternativas) de solução que foram apresentadas, discutidas e adotadas nos documentos institucionais que sistematizaram e formalizaram a PGETES.

Do mesmo modo, a agregação de elementos oriundos de propostas metodológicas de análise de viabilidade de políticas 12 ao modelo analítico de Kingdon 11 permite que se busque identificar facilidades e dificuldades, oportunidades e ameaças existentes no âmbito institucional e no contexto mais amplo. Assim, são apontadas restrições e possibilidades que podem ter influído na decisão e determinam as características da implementação da política.

No que tange à busca de evidências empíricas, as informações foram extraídas de documentos institucionais, notadamente relatórios de oficinas realizadas durante o processo de formulação da política, bem como as versões preliminares e a versão final do documento apre- sentado ao Colegiado de Gestão da SESAB. A leitura e análise do conteúdo dos documentos privilegiaram a identificação dos problemas existentes na área de recursos humanos, abarcando aspectos tanto quantitativos, relacionados com a disponibilidade e distribuição de pessoal das diversas categorias profissionais no SUS-Bahia, quanto qualitativos, referentes às deficiências verificadas na formação e na gestão de pessoas. Foram recortadas e sistematizadas informações relativas às propostas de encaminhamento e solução dos problemas identificados, tratando-se de registrar as contribuições dos diversos atores envolvidos.

Também foram utilizados registros da observação participante realizada por uma das autoras, que atuou como dirigente da Superintendência de Recursos Humanos da SESAB, órgão que conduziu o processo de formulação da política ao longo do período estudado. Tais registros foram feitos seguindo um roteiro previamente definido para a seleção e apreensão dos dados 16 . Os encontros eram precedidos de uma discussão com assessores da superintendente para definir aspectos importantes a serem observados durante a coordenação das oficinas e seminários realizados ao longo do período de elaboração da PGETES e constituíram fonte de informações utilizadas para compor o diário de campo da autora. Neste, foram privilegiados o mapeamento dos atores e suas posições diante dos problemas e propostas debatidas.

O processamento das informações foi feito levando-se em conta três grandes categorias de análise: o contexto, os atores e o processo decisório.

\section{Resultados}

A análise das informações produzidas ao longo do período estudado é apresentada em três momentos: descrição do contexto político estadual e do contexto político-institucional na SESAB; caracterização dos atores envolvidos; descrição e análise do processo de formulação da PGETES, formalizada no âmbito do SUS-Bahia em 2008.

\section{Contexto político}

O contexto político geral no qual se desenvolveu o processo de formulação da PGETES caracteriza-se pela mudança de direcionalidade na gestão pública estadual, a partir da eleição realizada em outubro de 2006. Nesta, saiu vitoriosa uma coligação liderada pelo Partido dos Trabalhadores, sob o comando de Jaques Wagner, governador eleito. 
O projeto de governo construído durante a campanha eleitoral começou a ser aperfeiçoado no período de transição, quando foram definidos os quadros dirigentes que assumiram, a partir de janeiro de 2007, a gestão das secretarias e demais órgãos do Governo do Estado. Esse processo configurou-se como uma mudança de grandes proporções, na medida em que a administração pública na Bahia permanecia há décadas sob comando de um mesmo grupo político, que, embora se mantenha firmemente ancorado nas estruturas de poder 17, vem sendo paulatinamente substituído nos cargos e funções governamentais.

Na SESAB, essa mudança significou a retomada dos princípios e diretrizes da Reforma Sanitária, os quais haviam pautado as iniciativas governamentais no setor durante o breve período de implantação do Sistema Unificado e Descentralizado de Saúde (SUDS), no final dos anos 80, no Governo Waldir Pires 4 . Já na década de 90, cenário de construção do SUS em todo o pais, particularmente a partir de 1993, o processo de implantação na Bahia sofreu as vicissitudes decorrentes do conflito de interesses e objetivos no interior das forças dirigentes, resultando em um atraso considerável em vários setores, inclusive na área de gestão do trabalho e da educação em saúde $18,19,20$. Desse modo, a primeira tarefa do grupo que se articulou em torno do novo Secretário de Saúde, Jorge Solla, foi proceder a um levantamento exaustivo de informações que permitiram uma primeira análise da situação existente no sistema público estadual. Identificou-se, assim, um conjunto de problemas que se constituíram no ponto de partida para a formulação do Plano dos 100 dias, em caráter emergencial.

Ao assumir a Secretaria de Saúde, em 2007, o secretário definiu sua equipe e desencadeou o processo de elaboração da Agenda Estratégica 2007 10, na qual já aparece como prioritária a área de recursos humanos. Esse setor estava sob responsabilidade da Superintendência de Recursos Humanos, criada durante a reforma administrativa realizada na SESAB em 2002. Faziam parte dessa superintendência quatro diretorias, responsáveis pelo planejamento de recursos humanos, administração de pessoal e a direção de cada uma das duas escolas que integram a rede de formação de pessoal do SUS-Bahia, a Escola de Formação Técnica Prof. Aristides Novis e a Escola de Saúde Pública Prof. Francisco Peixoto de Magalhães.

Uma das primeiras iniciativas da nova superintendente, nomeada pelo secretário, foi a redefinição da estrutura e das funções da diretoria de planejamento. Esta passou a se ocupar da gestão do trabalho e educação na saúde, ao mes- mo tempo em que constituiu um grupo técnico responsável pelo levantamento e sistematização de informações que possibilitassem a realização de um diagnóstico mais preciso acerca da problemática dos recursos humanos no âmbito do SUS estadual. O trabalho desse grupo técnico tornou-se o embrião do processo de formulação da PGETES, constituindo-se no Ator central, em torno do qual gravitou o conjunto de atores governamentais e não governamentais, caracterizados no item a seguir.

\section{Atores envolvidos na formulação da PGETES}

Seguindo os critérios propostos por Kingdon 11, cabe identificar, em primeiro lugar, os atores governamentais visíveis na cena política. No caso da PGETES, são o Secretário de Saúde e demais dirigentes da SESAB, que compunham o Colegiado de Gestão do SUS-Bahia, integrado pelos responsáveis pela condução dos principais órgãos do nível central: as cinco superintendências, a Assessoria de Planejamento, a Auditoria do SUS, a Direção do Fundo Estadual de Saúde, a Coordenadoria de Controle Interno, a Ouvidoria e a Direção Geral da SESAB.

De fato, o Colegiado de Gestão constituiu, durante o período estudado, o fórum privilegiado de tomada de decisões que se expressaram nos objetivos, diretrizes e estratégias adotadas ao longo da gestão, tendo sido sistematizadas em um conjunto de documentos institucionais. Destes, os mais importantes, sem dúvida, foram a Agenda Estratégica, atualizada anualmente, o Plano Estadual de Saúde, elaborado para o período 2008-2011, e as portarias emitidas pelo Secretário de Saúde.

As decisões relativas ao encaminhamento da política de desenvolvimento de recursos humanos incluíram, primeiramente, o enfrentamento das questões administrativas relacionadas com a contratação de pessoal em caráter emergencial para suprimento de cargos e funções que se encontravam vagos diante de resistências políticas corporativas às medidas de controle adotadas de imediato pelo Secretário. Um exemplo foi o rompimento do contrato com as cooperativas de profissionais de saúde que monopolizavam a oferta de força de trabalho em setores estratégicos.

Paralelamente, a Superintendência de Recursos Humanos constituiu um grupo técnico específico para aprofundar a análise da situação da força de trabalho em saúde no SUS, composto pelos quatro diretores e pelos coordenadores das áreas que compunham cada uma das diretorias. A composição do grupo técnico envolveu, além dos dirigentes e técnicos da SESAB, um 
conjunto de informantes-chave identificados ao longo do processo de análise da situação dos recursos humanos na saúde, a exemplo de membros da Comissão Intergestora Bipartite. Esse grupo técnico pode ser entendido como o "ator central" do processo de formulação da política (PGETES), apesar de a decisão propriamente dita ser atribuição do Colegiado de Gestão, a quem coube encaminhar as propostas apresentadas pelo grupo técnico às instâncias gestoras do SUS estadual: a Comissão Intergestores Bipartite e o Conselho Estadual de Saúde.

Além desses atores governamentais, participantes do processo de formulação da PGETES em virtude da posição que ocupavam na estrutura de poder do SUS estadual, cabe registrar que o processo contemplou o envolvimento de outros atores governamentais e não governamentais. Estes foram convidados a integrar o coletivo de participantes de reuniões e oficinas de trabalho realizadas ao longo dos quase dois anos em que o processo se desenvolveu.

Vale destacar que a formulação da PGETES ocorreu simultaneamente e de forma entrelaçada a outros processos de formulação de políticas no âmbito da SESAB, gerando o envolvimento de um conjunto de atores que eventualmente deram sua contribuição. Entre estes, distinguemse os participantes da 7a Conferência Estadual de Saúde, representantes dos gestores, trabalhadores e usuários do SUS, que discutiram, entre outras questões, a problemática dos recursos humanos 10.

Também cabe registrar que, nas reuniões e oficinas de trabalho realizadas para a elaboração do Plano Estadual de Saúde (PES) 2008-2011, entre estas as nove Oficinas Macrorregionais, que incluíram representantes dos 417 municípios do Estado da Bahia, foram abordadas as questões relativas à gestão do trabalho e educação na saúde 21 . Ao lado desses eventos, foram organizados seminários e oficinas especificamente voltadas para o aprofundamento da problemática da formação e capacitação de recursos humanos. Nessas ocasiões, foram recolhidas sugestões e recomendações de atores diversos, como os representantes das universidades públicas e particulares que desenvolvem cursos de graduação e pós-graduação na área de saúde, com as quais a Superintendência de Recursos Humanos, por meio das escolas, mantém parcerias.

Com isso, pode-se considerar que o processo de formulação da PGETES caracterizou-se pela confluência de um conjunto heterogêneo de debates que ocorreram em múltiplas arenas. O espaço privilegiado de sistematização dos problemas e das propostas foi constituído pelo grupo técnico da Superintendência de Recursos
Humanos, coordenado pelo Colegiado de Gestão deste órgão, que incluiu a superintendente e as quatro diretoras responsáveis pelos setores supracitados.

\section{Processo de formulação da PGETES: problemas e propostas}

O processo de formulação da PGETES contemplou dois momentos, que correspondem, no modelo analítico proposto por Kingdon 11, a duas "correntes" da política, a corrente dos problemas e a das alternativas de solução. De fato, a identificação de problemas na área de gestão do trabalho e educação na saúde não é um processo simples, desde que, no âmbito das pesquisas sobre o tema, coexistem múltiplos referenciais e abordagens teóricas, que se expressam, por vezes, em dificuldades operacionais no momento da definição dos problemas reais. No que diz respeito às soluções, ainda mais quando se trata de um processo que envolve vários atores, com interesses distintos, a formulação de propostas se torna um espaço de negociação e conflitos.

Isso, sem dúvida, ocorreu ao longo do conjunto de reuniões e oficinas que pontuaram o desenvolvimento do processo, evidenciando-se, por vezes, conflitos entre dirigentes que expressavam posições distintas com relação ao planejamento e à gestão da política de desenvolvimento de recursos humanos. A maioria desses conflitos opunha atores que tinham visão mais estratégica, voltada ao levantamento de problemas estruturais na área de recursos humanos no SUS, a dirigentes mais "pragmáticos", preocupados com $o$ atendimento de demandas de curto prazo.

Um exemplo desse conflito diz respeito à valorização diferencial atribuída pelos participantes ao planejamento de recursos humanos ou à gestão de pessoal. Os que defendem o planejamento se preocupam com a melhoria da qualidade da informação sobre o quantitativo de pessoal inserido no SUS-Bahia, sua distribuição territorial e institucional, a avaliação do seu desempenho, informações que servem de subsídios a um planejamento de médio e longo prazo. Os dirigentes mais preocupados com a gestão do trabalho, por seu turno, envolvem-se mais freqüentemente em debates acerca das alternativas de contratação e formas de sensibilização e comprometimento dos profissionais e trabalhadores com o SUS.

Outro exemplo de conflito refere-se ao posicionamento assumido por alguns participantes com relação aos fluxos decisórios, problematizando-se, às vezes, a relação entre a SESAB e o Ministério da Saúde, ou a relação entre a SESAB e os municípios. Cabe ressaltar que esses conflitos geralmente não se apresentam claramente, po- 
dendo aparecer sob a forma de polêmicas acerca do grau de prioridade dado a determinados problemas ou se dar na formulação das propostas de solução.

Também é possível identificar conflitos subjacentes pela análise do conteúdo dos debates travados nas oficinas, como, por exemplo, a alternativa de enfrentamento da problemática da escassez de quadros qualificados a exercerem funções gerenciais no nível regional e municipal, bem como a inadequação do perfil dos profissionais e trabalhadores que atuam na "ponta" do sistema. A resposta a estes problemas pode variar entre a elaboração de propostas mais amplas de profissionalização da gestão ou a qualificação técnico-gerencial de profissionais já inseridos na rede de serviço.

Apesar disso, o processo de formulação da PGETES confluiu para a construção de um amplo consenso em torno dos problemas prioritários, sendo mais difícil de se chegar a um acordo com relação às alternativas de encaminhamento e solução para eles. Isso se deve ao fato de que se enfrentava a incerteza quanto ao horizonte temporal em que foi pensada a política, optando-se por formulá-la considerando a possibilidade de implementação no tempo político de quatro anos, ainda que os objetivos apontassem para além desse prazo.

Assim, no que tange à identificação dos problemas, obteve-se um amplo consenso, conseguindo-se distinguir os problemas relativos às características dos profissionais e trabalhadores inseridos no SUS-Bahia, em termos de perfil e grau de qualificação, daqueles relacionados às condições de trabalho a que estão sujeitos. Ao lado disso, foram identificados os problemas específicos relativos à atuação da SESAB sobre esse contingente de trabalhadores, problemas vinculados tanto à gestão do trabalho, quanto à intervenção sobre a formação e qualificação dos trabalhadores em atividade. Também foram identificados os problemas específicos da própria Superintendência de Recursos Humanos (Tabela 1).

Com base nos problemas identificados, foram sistematizados quatro, os quais ingressaram na Agenda Estratégica da SESAB em 2007-2008. Das características dos trabalhadores, foi priorizada a problemática da "precarização das relações de trabalho: vínculos e condições”, que afeta a grande maioria dos trabalhadores do SUS, inclusive em áreas estratégicas, como é o caso da atenção básica, e nos serviços hospitalares de urgência/emergência. No que diz respeito às ações sob responsabilidade da Superintendência de Recursos Humanos da SESAB, foi destacada a "fragilidade nas ações, processos, sistemas e instrumentos de gestão de pessoas e do trabalho em saúde”, problema afetado pela deficiência do sistema de gerenciamento do funcionalismo público no Estado da Bahia. Também se priorizou a "inadequação dos processos pedagógicos, administrativos e financeiros para a formação e desenvolvimento dos trabalhadores às necessidades do SUS-Bahia”, problema que reflete a necessidade de atualização permanente das equipes envolvidas com a elaboração de projetos político-pedagógicos e com a implementação de ações educativas na rede de instituições de ensino superior e médio. Finalmente, foi priorizada a "incipiência da PGETES em saúde para o SUSBahia”, problema que galvanizou os esforços do conjunto da equipe, na medida em que se considerou que sua solução pressupunha a participação dos vários setores da SESAB responsáveis pela gestão da atenção à saúde, em seus vários níveis. Esse problema não só envolveu os municípios, mediante o Conselho de Secretários $\mathrm{Mu}$ nicipais de Saúde e as universidades (públicas e privadas) que operam no estado, como também promoveu uma articulação intersetorial com outras secretarias do estado e o Ministério Público. Como se pode observar, trata-se de um problema cuja solução atravessa o conjunto do sistema, tendo, portanto, um grande potencial de promover mudanças estruturais, caso venha a ser enfrentado adequadamente.

Com base nisso, foi desencadeado o processo de elaboração das alternativas, as quais foram sistematizadas em um documento apresentado sucessivamente nas instâncias gestoras do SUSBahia estadual, sendo aprovado em julho de 2008. A partir daí, o Colegiado de Gestão da Superintendência de Recursos Humanos e os técnicos das diretorias envolvidos no processo de elaboração do Plano Estadual de Saúde levaram aos debates as propostas da PGETES, as quais foram incorporadas na pauta das oficinas e, posteriormente, constituíram-se no Módulo Operacional de Gestão do Trabalho e Educação na Saúde do PES 2008-2011.

Cabe ressaltar que o debate no âmbito dessas oficinas contemplou a análise de viabilidade de implantação das ações propostas. Foram aprecidadas a disponibilidade de recursos financeiros com base na revisão dos projetos integrantes do Plano Plurianual do Estado da Bahia 2007-2010 e a disponibilidade de capacidade gerencial e técnico-operacional na Escola Estadual de Saúde Pública e na Escola Técnica do SUS-Bahia, órgãos responsáveis pela articulação de parcerias com instituições formadoras de pessoal em saúde.

A Tabela 2 sintetiza os principais objetivos e ações estratégicas da PGETES incorporadas ao PES 2008-2011. Como se pode constatar, os 
Problemas identificados na área de recursos humanos do SUS-Bahia, Brasil, 2007.

\begin{tabular}{|c|c|c|c|}
\hline $\begin{array}{l}\text { Características dos trabalhadores } \\
\text { e das condições de trabalho no } \\
\text { SUS-Bahia }\end{array}$ & $\begin{array}{l}\text { Problemas na área de gestão } \\
\text { do trabalho e administração de } \\
\text { recursos humanos }\end{array}$ & $\begin{array}{l}\text { Problemas na área de formação e } \\
\text { qualificação de pessoal }\end{array}$ & $\begin{array}{c}\text { Problemas na estrutura } \\
\text { e funcionamento da } \\
\text { Superintendência de Recursos } \\
\text { Humanos da SESAB }\end{array}$ \\
\hline $\begin{array}{l}\text { Perfil profissional inadequado } \\
\text { para as necessidades dos vários } \\
\text { processos de trabalho da rede } \\
\text { SUS-Bahia. }\end{array}$ & $\begin{array}{c}\text { Desconhecimento das } \\
\text { necessidades referentes à força } \\
\text { de trabalho para o SUS-Bahia e } \\
\text { sistema de informação ineficiente } \\
\text { na produção de dados para } \\
\text { tomada de decisões. }\end{array}$ & $\begin{array}{l}\text { Existência de processos } \\
\text { de educação na saúde não } \\
\text { referenciados aos princípios, } \\
\text { diretrizes e necessidades } \\
\text { do SUS-Bahia. }\end{array}$ & $\begin{array}{l}\text { Inadequação da estrutura física, } \\
\text { funções, sistemas, processos } \\
\text { de trabalho e equipamentos da } \\
\text { Superintendência às necessidades } \\
\text { do SUS. }\end{array}$ \\
\hline $\begin{array}{l}\text { Força de trabalho pouco } \\
\text { qualificada, principalmente nível } \\
\text { técnico-administrativo, para o } \\
\text { desempenho das novas funções do } \\
\text { SUS-Bahia. }\end{array}$ & $\begin{array}{c}\text { Inexistência de parâmetros e } \\
\text { instrumentos normativos relativos } \\
\text { à administração dos trabalhadores } \\
\text { da saúde que atendam às } \\
\text { necessidades do SUS. }\end{array}$ & $\begin{array}{l}\text { Inadequação da regulação dos } \\
\text { processos educativos em suas } \\
\text { várias dimensões (pedagógicas, } \\
\text { administrativas e financeiras). }\end{array}$ & $\begin{array}{l}\text { Ausência ou desativação de } \\
\text { estruturas, espaços formais, } \\
\text { representativos e descentralizados } \\
\text { de gestão da educação } \\
\text { e do trabalho. }\end{array}$ \\
\hline $\begin{array}{l}\text { Relações de trabalho precárias: } \\
\text { vínculos e condições. }\end{array}$ & $\begin{array}{l}\text { Inexistência de ferramentas } \\
\text { informatizadas que possibilitem a } \\
\text { gestão em rede de pessoas e de } \\
\text { postos de trabalho. }\end{array}$ & $\begin{array}{l}\text { Pouca diversificação e limitada } \\
\text { oferta de cursos da Escola de } \\
\text { Formação Técnica em Saúde. }\end{array}$ & \\
\hline $\begin{array}{l}\text { Desconhecimento por parte dos } \\
\text { trabalhadores acerca do SUS, } \\
\text { do seu papel na instituição e } \\
\text { das funções da secretaria como } \\
\text { gestora estadual do SUS. }\end{array}$ & $\begin{array}{l}\text { Distorção das formas de } \\
\text { concessões de gratificações, } \\
\text { direitos e vantagens. }\end{array}$ & $\begin{array}{c}\text { Falta de regulamentação/ } \\
\text { credenciamento da Escola Estadual } \\
\text { de Saúde Pública frente aos órgãos } \\
\text { reguladores da educação. } \\
\text { Baixa incorporação tecnológica } \\
\text { que permita descentralizar, } \\
\text { ampliar e capilarizar processos de } \\
\text { educação necessários para o SUS. } \\
\text { Insuficiência do uso da rede de } \\
\text { unidades como espaço de ensino } \\
\text { e aprendizagem para profissionais } \\
\text { do SUS. }\end{array}$ & \\
\hline
\end{tabular}

SESAB: Secretaria Estadual de Saúde da Bahia.

Fonte: Superintendência de Recursos Humanos da SESAB.

objetivos contemplam a solução dos problemas priorizados, enquanto as ações estratégicas propostas tentam manter coerência com as diretrizes políticas e organizacionais assumidas pela gestão estadual com relação ao SUS. Essas ações enfatizam o fortalecimento das estruturas regionais e locais responsáveis pela gestão do trabalho e assumem a responsabilidade constitucional pela ordenação da formação de recursos humanos em saúde, consolidando a atribuição conferida às escolas técnica e de saúde pública do SUS de capacitarem e titularem profissionais e trabalhadores de saúde.

O conjunto dessas diretrizes e ações estratégicas adotadas foi pactuado, aprovado e ampla- mente divulgado sob o lema "o SUS é uma escola”, conferindo direcionalidade aos programas e projetos atualmente em execução e confluindo para a proposta, recentemente aprovada, da Universidade Aberta do SUS, já em fase de implementação no estado.

\section{Discussão}

A análise do processo de formulação da PGETES permite que se coloquem algumas questões para reflexão, no que diz respeito à aplicabilidade e potência tanto do modelo analítico proposto, quanto dos conhecimentos produzidos acerca 
Política de Gestão do Trabalho e Educação na Saúde (PGETES): objetivos e ações estratégicas. SUS-Bahia, Brasil, 2008.

\section{Objetivos}

Implementar mecanismos descentralizados e regionalizados de gestão do trabalho e da educação permanente.

Implementar alternativas gerenciais que permitam a garantia do cumprimento dos direitos trabalhistas dos servidores da saúde e a melhoria das suas condições de trabalho e remuneração.

Ordenar o processo de formação e qualificação de pessoal de nível médio em saúde.

Ordenar o processo de formação e qualificação de pessoal de nível superior em saúde.

\section{Ações Estratégicas}

- Implantação da câmara técnica estadual da gestão do trabalho e da educação permanente em saúde junto à Comissão Intergestores Bipartite.

- Apoio aos municípios para a estruturação da gestão do trabalho e de educação permanente em saúde.

- Implementação do HumanizaSUS no Estado da Bahia.

- Desenvolvimento e implantação do sistema de informação sobre a força de trabalho em saúde no SUS-Bahia.

- Implementação de Mesa de Negociação Permanente do SUS-Bahia.

- Realização do diagnóstico da força de trabalho do SUS-Bahia.

- Implantação dos núcleos descentralizados de gestão de pessoas, por meio da reestruturação das coordenações de recursos humanos, das unidades administrativas regionais (DIRES) e unidades de saúde que compõem a rede própria de serviços da SESAB (hospitais e centros de referência).

- Dimensionamento da força de trabalho da SESAB.

- Implementação do Plano de Cargos, Carreiras e Vencimentos (PCCV) da SESAB. Suprimento das lacunas de pessoal da SESAB por meio de processo seletivo simplificado e/ ou concurso público.

- Formação e desenvolvimento de pessoal no SUS-Bahia.

- Formulação e avaliação de diretrizes para a formação técnica na Bahia.

- Capacitação técnico-político-pedagógica para docentes que atuam nos cursos de formação técnica em saúde.

- Apoio às equipes técnicas das secretarias municipais de saúde para a implementação dos processos educativos.

- Definição e desenvolvimento de pesquisas na área de formação técnica de trabalhadores da saúde.

- Organização da rede de educação-trabalho envolvendo as escolas técnicas de saúde.

- Regulação dos campos de prática.

- Implantação, na Bahia, da Escola Técnica Aberta do Brasil (e-TEC Brasil).

- Organização das Redes Regionais de Integração Educação-Trabalho.

- Estruturação do sistema de informação para planejamento, execução, monitoramento e avaliação das ações de educação permanente no SUS-Bahia.

- Implantação, implementação e publicização do programa Universidade Aberta do SUS.

- Apoio à implementação das diretrizes curriculares com vistas ao reordenamento da formação e qualificação profissional de saúde.

- Apoio à implementação das diretrizes curriculares com vistas ao reordenamento da formação e qualificação profissional de saúde.

- Reordenamento, monitoramento e avaliação dos programas de residências em saúde no SUS-Bahia.

- Qualificação dos gestores e trabalhadores do SUS-Bahia, na perspectiva da educação permanente, de forma multicêntrica, regionalizada e descentralizada.

- Implantação do observatório de estudos e pesquisas em gestão do trabalho e da educação permanente em saúde.

- Implementação do projeto de educação a distância do SUS-Bahia como estratégia de ampliação e democratização do acesso a processos educativos e de comunicação.

SESAB: Secretaria Estadual de Saúde da Bahia.

Fonte: Plano Estadual de Saúde 2008-2011. 
das facilidades e dificuldades encontradas para o desenvolvimento da PGETES, não só no SUSBahia, mas também em outros estados do país.

Como se depreende do exposto no item anterior, o contexto em que se desenvolveu a formulação da Política configurou uma "janela de oportunidade" 11 para a decisão dos atores envolvidos, uma vez que, simultaneamente, contemplou um balanço favorável à inclusão da problemática dos recursos humanos na agenda estratégica da gestão estadual e permitiu a organização de um processo participativo, que contribuiu para a obtenção do consenso acerca dos problemas a serem enfrentados.

Os processos desencadeados consistiram em aprendizado institucional 22, já que a busca pelo estabelecimento do consenso em torno dos princípios norteadores das propostas de ação da SESAB nesta área foi a tônica da Superintendência de Recursos Humanos durante o período estudado. Sendo assim, é importante destacar o consenso que se alcançou entre o grupo dirigente com relação à adoção da educação permanente, entendida como estratégia de reconfiguração do modus operandi dos trabalhadores de saúde, ou seja, estratégia para o fortalecimento dos processos de mudança na gestão e na organização da prestação de ações e serviços, eixos principais da política de saúde adotada na atual gestão.

No que diz respeito à possibilidade de implantação, vale a pena registrar que, ainda durante o processo de formulação da PGETES, foram feitos investimentos na melhoria da infraestrutura e na composição do quadro gerencial e docente das escolas do SUS. Essas ações objetivaram fortalecer uma inteligência estratégica, capaz de redirecionar não só os programas de formação e capacitação profissional com ênfase na qualificação dos trabalhadores envolvidos nas redes assistenciais, mas também as linhas de cuidado dirigidas a grupos populacionais e problemas prioritários, incorporando novas estratégias pedagógicas, como educação à distância.
Nessa perspectiva, foram desencadeados vários projetos contemplando a transformação dos espaços de trabalho em áreas de formação e educação permanente, por meio da reorganização dos cenários de práticas/estágios na rede SUS e do envolvimento das equipes e dos trabalhadores/ técnicos como agentes multiplicadores da política. Essa mudança implica, também, o estímulo à produção de estudos e pesquisas sobre a problemática da gestão do trabalho e da educação em saúde no âmbito do SUS.

O estudo de caso aqui apresentado traz elementos que subsidiam o debate 23,24 sobre a necessidade de se consolidar um instrumental que viabilize processos de formulação e implementação de políticas nos diferentes estados e municípios brasileiros, cujas fragilidades foram demonstradas por Pierantoni et al. 23 em estudo sobre a capacidade gestora de Secretarias $\mathrm{Mu}$ nicipais de Saúde. Os autores chamam atenção para o fato de que o planejamento das ações de recursos humanos não está incorporado na prática dos dirigentes da área.

Finalmente, vale ressaltar que a experiência desenvolvida na SESAB no período estudado aponta para a possibilidade de se avançar no processo de institucionalização do planejamento e gestão de políticas públicas 25 , com o fortalecimento da capacidade de formulação e implementação de políticas, como foi o caso da PGETES. Esse processo se insere no conjunto das iniciativas desencadeadas na gestão 2007-2008 e faz parte dos esforços indicados por Pierantoni et al. 26 como necessários para a consolidação da área de recursos humanos.

Com isso, espera-se que outros estudos possam ser realizados, contemplando, por exemplo, a análise do processo de implementação da PGETES na ótica dos vários atores que participaram do processo, bem como pesquisas avaliativas dos efeitos das ações desencadeadas a partir da política sobre as práticas de gestão e atenção à saúde da população. 


\section{Resumo}

A construção do SUS tem colocado um conjunto de desafios aos dirigentes e técnicos do setor, entre os quais as questões relativas à gestão do trabalho e a educação permanente dos trabalhadores da saúde, tendo em vista as limitações financeiras, políticas e organizacionais do processo de mudança do modelo de atenção à saúde. O objetivo deste trabalho é analisar o processo de formulação da Política de Gestão do Trabalho e Educação na Saúde, desenvolvido na Secretaria Estadual de Saúde da Bahia. Utiliza como referencial a teoria do ciclo da política pública. As informações analisadas foram extraídas de documentos institucionais e registros da observação participante realizada por uma das autoras. Os resultados contemplam o mapeamento dos atores governamentais e não governamentais que participaram do processo. A análise evidencia o conjunto de problemas existentes no SUS-Bahia com respeito à gestão do trabalho e ao perfil dos trabalhadores de saúde, o que foi tomado como ponto de partida para definição das prioridades na Agenda Estratégica e no Plano Estadual de Saúde 2008-2011.

Políticas Públicas de Saúde; Pessoal de Saúde; Sistema Único de Saúde

\section{Colaboradores}

I. C. M. Pinto participou da realização da pesquisa, análise e interpretação dos dados e redação do texto. C. F. Teixeira colaborou na revisão e redação final do texto.

\section{Agradecimentos}

Ao conjunto de sujeitos que participaram do processo de formulação da Política de Gestão do Trabalho e Educação na Saúde, especialmente ao grupo de trabalho responsável pela sistematização do documento.

\section{Referências}

1. Levcovitz E, Lima L, Machado C. Política de saúde nos anos 90: relações intergovernamentais e o papel das Normas Operacionais Básicas. Ciênc Saúde Coletiva 2001; 6:269-91.

2. Goulart, F.A.A. Esculpindo o SUS a golpes de portaria: considerações sobre o processo de formulação das NOBs. Ciênc Saúde Coletiva 2001; 6:292-8.

3. Teixeira CF. Promoção e vigilância da saúde no SUS: desafios e perspectivas. In: Teixeira CF, organizador. Promoção e vigilância da saúde. Salvador: Centro de Educação Permanente em Saúde, Instituto de Saúde Coletiva, Universidade Federal da Bahia; 2002. p. 101-25.

4. Paim JS. Modelos assistenciais: reformulando o pensamento e incorporando a proteção e a promoção da saúde. In: Paim JS, organizador. Saúde, política e reforma sanitária. Salvador: Centro de Educação Permanente em Saúde, Instituto de Saúde Coletiva, Universidade Federal da Bahia; 2002. p. 367-81.
5. Ministério da Saúde. Diretrizes operacionais dos pactos pela vida, em defesa do SUS e de gestão. Brasília: Ministério da Saúde; 2006.

6. Costa NR. O Banco Mundial e a política social nos anos 90. In: Costa NR, Ribeiro JM, organizadores. Política de saúde e inovação institucional. Rio de Janeiro: Escola Nacional de Saúde Pública, Fundação Oswaldo Cruz; 1996. p. 13-29.

7. Almeida C. Reforma sanitária brasileira: um trajeto de mudanças. Rio de Janeiro: Escola Nacional de Saúde Pública, Fundação Oswaldo Cruz; 1993. (Série Estudos, 1).

8. Mendes EV. Uma agenda para a saúde. São Paulo: Editora Hucitec; 1996.

9. Paim JS. Reforma sanitária e municipalização. Saúde Soc 1992; 1:29-48.

10. Secretaria Estadual de Saúde da Bahia. Relatório da 7a Conferência Estadual de Saúde. Salvador: Secretaria Estadual de Saúde da Bahia; 2007. 
11. Kingdon JW. Agendas, alternatives and public policies. Boston: Addison-Wesley Longman; 1995.

12. Teixeira CF, Ribeiro M. Carvalho M. O processo de formulação da política de saúde da população negra em Salvador 2005-2006. Relatório de pesquisa. Salvador: Instituto de Saúde Coletiva, Universidade Federal da Bahia; 2009.

13. Viana AL. Abordagens metodológicas em políticas públicas. Rev Adm Pública 1996; 30:5-43.

14. Baumgarter FR, Jones BD. Attention, boundary effects and large-scale policy change in air transportation policy. In: Rochefort D, Cobb R, editors. The politics of problem definition: shaping the policy agenda. Lawrence: University Press of Kansas; 1995. p. 50-66.

15. Pinto ICM Ascensão e queda de uma questão na agenda governamental: o caso das organizações sociais da saúde na Bahia [Tese de Doutorado]. Salvador: Núcleo de Pós-graduação em Administração, Universidade Federal da Bahia; 2004.

16. Minayo MC. O desafio do conhecimento: pesquisa qualitativa em saúde. São Paulo: Editora Hucitec/ Rio de Janeiro: ABRASCO; 1992.

17. Dantas Neto PF. O Carlismo para além de ACM: estratégias adaptativas de uma elite política estadual. In: Souza C, Dantas Neto PF, organizadores. Governo, políticas públicas e elites políticas nos estados brasileiros. v. 1. Rio de Janeiro: Revan; 2006. p. 247-86.

18. Molesini JA. Municipalização da saúde na Bahia: estudo exploratório da implementação da NOB 01/93 [Dissertação de Mestrado]. Salvador: Instituto de Saúde Coletiva, Universidade Federal da Bahia; 1999.
19. Guimarães MCL. Descentralização da saúde, interesses e conflitos decisórios: o processo de decisão nas instâncias colegiadas estaduais, Bahia, 19931998 [Tese de Doutorado]. Salvador: Escola de Administração, Universidade Federal da Bahia; 2000.

20. Coelho TCB. O processo político de gestão em uma instituição hipercomplexa do Sistema Único de Saúde [Tese de Doutorado]. Salvador: Instituto de Saúde Coletiva, Universidade Federal da Bahia; 2002.

21. Abreu de Jesus W, Teixeira CF. Planejamento estadual do SUS: o caso da Secretaria de Saúde do Estado da Bahia. Ciênc Saúde Coletiva 2010; 15:2383-93

22. Senge P. O novo trabalho do líder: construindo organizações que aprendem. In: Starkey K, organizador. Como as organizações aprendem. São Paulo: Editora Futura; 1997. p. 342-76.

23. Pierantoni CR, Varella TC, França T. Recursos humanos e gestão do trabalho em saúde: da teoria para a prática. In: Barros AFR, Santana JP, Santos Neto PM, organizadores. Observatório de recursos humanos em saúde no Brasil: estudos e análises. v. 2. Brasília: Ministério da Saúde; 2004. p. 51-70.

24. Nogueira RP. Problemas de gestão e regulação do trabalho no SUS. Serv Soc Soc 2006; 87:147-62.

25. Paim JS, Teixeira CF. Configuração institucional e gestão do Sistema Único de Saúde: problemas e desafios. Ciênc Saúde Coletiva 2007; 12:1819-29.

26. Pierantoni CR, Varella TC, Santos MR, França T, Garcia AC. Gestão do trabalho e da educação em saúde: recursos humanos em duas décadas do SUS. Physis (Rio J.) 2008; 18:685-704.

Recebido em 22/Ago/2010

Versão final reapresentada em 19/Fev/2011 Aprovado em 22/Jun/2011 